Journal of the British Society for Phenomenology, Vol. 37, No. 3, October 2006

\title{
THE DIFFERENTIAL POINT OF VIEW OF THE INFINITESIMAL CALCULUS IN SPINOZA, LEIBNIZ AND DELEUZE SIMON DUFFY
}

In Hegel ou Spinoza, ${ }^{1}$ Pierre Macherey challenges the influence of Hegel's reading of Spinoza by stressing the degree to which Spinoza eludes the grasp of the Hegelian dialectical progression of the history of philosophy. He argues that Hegel provides a defensive misreading of Spinoza, and that he had to "misread him" in order to maintain his subjective idealism. The suggestion being that Spinoza's philosophy represents, not a moment that can simply be sublated and subsumed within the dialectical progression of the history of philosophy, but rather an alternative point of view for the development of a philosophy that overcomes Hegelian idealism. Gilles Deleuze also considers Spinoza's philosophy to resist the totalising effects of the dialectic. Indeed, Deleuze demonstrates, by means of Spinoza, that a more complex philosophy antedates Hegel's, which cannot be supplanted by it. Spinoza therefore becomes a significant figure in Deleuze's project of tracing an alternative lineage in the history of philosophy, which, by distancing itself from Hegelian idealism, culminates in the construction of a philosophy of difference.

It is Spinoza's role in this project that will be demonstrated in this paper by differentiating Deleuze's interpretation of the geometrical example of Spinoza's Letter XII (on the problem of the infinite) in Expressionism in Philosophy, Spinoza, ${ }^{2}$ from that which Hegel presents in the Science of Logic. ${ }^{3}$ Both Hegel and Deleuze each position the geometrical example at different stages in the early development of the differential calculus. By demonstrating the relation between "the differential point of view of the infinitesimal calculus" and the differential calculus of contemporary mathematics, Deleuze effectively bypasses the methods of the differential calculus which Hegel uses to support the development of the dialectical logic.

\section{Letter XII and "the Problem of the Infinite"}

The relation of implication and involvement which ties the finite to the infinite, "for an adequate knowledge" (Macherey, 163), is determined by Spinoza in Letter XII where he develops "the problem of the infinite":

Everyone has always found the problem of the Infinite very difficult, indeed insoluble. This is because they have not distinguished between what is infinite as a consequence of its own nature, or by the force of its definition, and what has no bounds, not indeed by the force of its essence, but by the force of its cause. And also because they have not distinguished between what is called infinite because it has no limits and that whose parts we cannot explain or equate with any number, though we know its maximum and minimum. Finally, they have not 
distinguished between what we can only understand, but not imagine, and what we can also imagine". ${ }^{4}$

In order to illustrate the distinctions involved in the "problem of the infinite", Spinoza proposes for our reflection an example from geometry, to which Hegel makes reference on many occasions:

... all the inequalities of the space between two circles, $\mathrm{A}$ and $\mathrm{B}$, and all the variations which the matter moving in it must undergo, exceed every number. That is not inferred from the excessive size of the intervening space. For however small a portion of it we take, the inequalities of this small portion will still exceed every number. Nor is it inferred because, as happens in other cases, we do not know its maximum and minimum. For we know both in this example of ours: $\mathrm{AB}$ is the maximum and $\mathrm{CD}$ is the minimum. Instead it is inferred simply from the fact that the nature of the space between two non-concentric circles does not admit anything of the kind"(Let. XII).

The example that Spinoza develops (Figure 1) consists of two nested nonconcentric circles, and the two extreme orthogonal distances between them, $\mathrm{AB}$ and $\mathrm{CD}$.

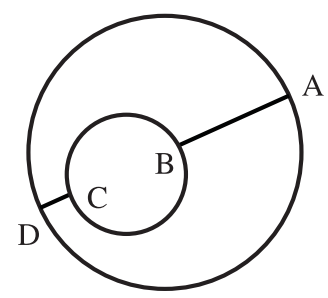

Figure 1

It allows Spinoza to present the relation between the finite and the infinite as follows: that

some things are infinite by their nature and cannot in any way be conceived to be finite, that others [are infinite] by the force of the cause in which they inhere, though when they are conceived abstractly they can be divided into parts and regarded as finite, and that others, finally, are called infinite, or if you prefer, indefinite, because they cannot be equated with any number, though they can be conceived to be greater or lesser. For if things cannot be equated with a number, it does not follow that they must be equal. This is manifest enough from the example adduced (Let. XII).

In the case of the double figure of non-concentric circles, what Spinoza suggests is that the variation of distances included between the external circle and the internal circle, not able to be determined by any number, is infinite, "or if you prefer, indefinite", even though it is understood within certain limits, that is, within the two circumferences and the greatest and least orthogonal distance, which function as thresholds or limits, as in a maximum and a minimum. 
Infinite Actu and the "Bad Infinite".

When Hegel makes reference to this geometrical example in the Science of Logic and the History of Philosophy he speaks only of the "space" interposed between the two circumferences. In the History of Philosophy he glosses Letter XII as follows: "The inequalities of the space [die ungleichen Abstände des Raumes] between $\mathrm{AB}$ and $\mathrm{CD}$ exceed every number; and yet the space which lies between is not so very great". ${ }^{5}$ The attempt to determine all of the spaces would be to constitute an infinite series, an infinity of unequal spaces. Hegel, however, argues that "Spinoza rejects" such a mathematical conception of the infinite "which represents it as an amount or as a series which is not completed", since, for Hegel, "the determination of magnitude contained in [such an unlimited series] cannot at the same time be represented as a quantum". ${ }^{6}$ According to Hegel, any attempt to divide a continuous variation, included between a minimum and a maximum, into parts in order to reconstitute the variation by a number will result in a false infinite, since the variation, which is continuous, can not be divided and extrapolated in this way, that is, it can not be determined numerically. What is discovered is a magnitude that does not equal any quantum or number, and which is determined rather as exceeding all number, as expressed in statements by Spinoza such as "and so on to infinity" ${ }^{7}$ Hegel argues that this non-numerical infinite is "there to hand, circumscribed, affirmative, actual and present in that plane as a complete space between the two circles". Hegel describes this circumscribed infinite as "philosophic infinity, that which is infinite actu" (LHP 262). Hegel designates the relation of substance to its affections by the concept of the "actual infinite", which is the realisation of the cause in its effects, that is, the actual infinite is the realisation in the affections, or the finite, of infinite substance. "It is an infinite which is not given in an unlimited series [as the bad infinite], but [rather] all at once: it is this [actual] infinite which is present in a limited reality, such as that included between a minimum and a maximum" (Macherey, 171). The actual infinite "is not beyond, but actually present and complete", as bounded within this space. ${ }^{8}$

The affirmation by Spinoza of the existence of an actual infinite expresses the implication and envelopment of the infinite in the finite. According to Hegel, it would be a manifest contradiction to assign number to the actual infinite since this is what distinguishes it from the bad infinite; the bad infinite being the idea of the actual infinite from the point of view of the finite. The idea of the bad infinite ignores the actual infinite, or "mistakes it with the idea of an unlimited series which excludes the possibility of an actual infinite" (Macherey, 171).

Hegel argues that Spinoza's concept of the actual infinite "might have been better expressed as "the negation of negation" (LHP 262). The finite is primarily negated by its opposite, the infinite, in the form of the bad infinite, since it is an 
infinite conceived from the point of view of the finite as an unlimited series. The bad infinite remains defective since the only negation with which it is affected is the primary negation of the finite. The bad infinite itself must then be negated, in order for the finite to realise itself as actually infinite. The negation of the bad infinite is therefore determinative of the actual infinite.

When Spinoza introduces the concept of the actual infinite in Letter XII from the point of view of the seventeenth-century mathematician, contrary to Hegel's understanding, mathematicians are presented as being on the side of philosophers. Spinoza argues that mathematicians "do not infer that ... [things which cannot be equated with any number] ... exceed every number because of the multiplicity of their parts", as Hegel suggests mathematicians do. Spinoza argues that mathematicians "infer" rather that "the nature of [such things] cannot admit number without a manifest contradiction" (Let. XII). With this statement Spinoza suggests that "no Infinite is explained in terms of the multitude of its parts; and that, if every Infinite escapes number, it is not because it contains too many parts, but because, by nature, it is not expressible by it". ${ }^{9}$ As a consequence, the introduction of a relation of contradiction as a means of determining the concept of a non-numerical, or actual, infinite can be avoided. Rather than acknowledging the consequences of this statement, Hegel reads it solely as a statement about the distinction between two infinities, the bad or mathematical infinite, and the actual or philosophic infinite. According to Hegel, the mathematicians rely on a concept of number in order to determine the concept of the infinite as the greatest of all numbers, or the indefinite or unlimited. This admission of number in the determination of the infinite enables Hegel to establish a vigorous contrast between the mathematical infinite and the philosophic infinite. The concept of a nonnumerical, philosophic or actual infinite can only be conceived in a relation of contradiction to a concept of the infinite that does admit number, namely the bad or mathematical infinite.

Hegel thus interprets Spinoza's presentation of the mathematical infinite in Letter XII as a bad infinite which is in a relation of contradiction with the actual or philosophic infinite, an interpretation which is contrary to Spinoza's actual remarks. According to Hegel, the bad infinite functions only as the immediate contrary of the finite, and is abrogated in the name of the finite realised as actually infinite. "As soon as the cause has something else opposed to it - the effect - finitude is present; but here this something else is at the same time abrogated and it becomes once more the cause itself. The affirmative is thus negation of negation" (LHP 262). The infinite, when opposed to the finite, is conceived as the bad infinite, which is then sublated and subsumed in the actual infinite, that is, the finite realises itself as actually infinite. This is how Hegel resolves the relation of the infinite to the finite from the point of view of his interpretation of Spinoza. 
This explanation by Hegel of "the problem of the infinite", in relation to the text of Spinoza, renders superfluous the particularities of the geometrical example as exposed by Spinoza. Macherey argues that, as far as Hegel is concerned, every finite mode "expresses an infinity, which it formally envelops ... as its cause" (Macherey 171) - for example, the space included between the two circles, or, to take another example put forward by Hegel, the infinity of points included in a segment of a line. This however ignores the essential fact that the two circles are non-concentric.

Hegel does not distinguish between, on the one hand, the expression of the circumference of circle A as a maximum and that of circle B as a minimum, and, on the other hand, the expression of a maximum and a minimum as the greatest and smallest distances between the circumference of circle A and circle B. The "maximum and minimum" serve Hegel simply as a means for defining the finite as a contained space or limited distance. Such an interpretation does not account for the specificity of presenting non-concentric circles in the example, since the same reasoning would hold if the distances which separate the two circumferences were all equal. It is therefore evident that "the two circles could not be concentric, for in this case there would be no inequalities among the segments" ${ }^{10}$ Macherey argues that "if Spinoza had only wanted ... to represent the idea of a finite quantum which entails nevertheless an infinity of parts [which] exceeds all assignable number" (Macherey, 172), he would not have had need of the precision that the two circles are nonconcentric. Hegel therefore neglects something that is essential to the reasoning proposed by Spinoza. This simplification by Hegel renders inevitable the reduction of the infinity between the circles to a simple unlimited series represented by the idea of the bad infinite, which is contradictory to the nature or essence of the finite thing, and which when negated is abrogated in the expression of it as actually infinite.

\section{The Problem of the "Bad Infinite".}

Macherey contends that, contrary to this Hegelian argument, things are not so simple. The reestablishment of a clear separation between the infinite and the finite is not sufficient to address what Macherey considers to be the tendency of the imagination to represent the infinite in the finite as the 'bad infinite', but merely begs the question of that tendency. Given that the relation of opposition between the finite and the infinite imposed by the dialectical logic springs from the negation of the infinite by the finite, Gueroult argues that "far from causing their opposition to vanish by suppressing" the expression of the infinite in favour of the expression of the finite, "the imagination transforms it into an irremediable conflict, for the infinite, wrongly understood" from the point of view of the imagination, "subsists no less truly in things, and its negation", again by the imagination, "amounts to 
nothing other than to confer upon it the properties of the finite. Thus, refusing to submit itself to violation, [the infinite] ... affirms its presence ... by causing to arise, from the depths of the alleged solutions dictated by the imagination, the fundamental absurdity which is at their source"(210). The infinite refuses to submit itself to, that is, it negates, the absurdity of the imaginary idea of the infinite as the bad infinite, the alleged solution dictated by the imagination, or the primary negation of the infinite by the finite. Therefore the establishment of the actual infinite is dependent on the irremediable imaginary conflict.

Following on from this argument presented by Gueroult, Macherey contends that Hegel's argument "therefore repeats the point of view of the imagination from which it seeks to distinguish itself" (172). What is neglected by Hegel is "that essential character of the finite", which, to return to the distinctions of the problem of the infinite presented by Spinoza in Letter XII, is "infinite by the force of its cause", that which, Macherey contends, is peculiar to all the modes, whether perceived to be infinite (unlimited) or finite (limited). "The variation of distances included between the two non-concentric circles is also infinite, not in itself since it is limited, but as an affection of substance which expresses itself in it as the cause in its effect" (170), that is, as the positive immanent expression of substance in its affections.

Macherey re-presents the distinctions of the "problem of the infinite" as being between that which is infinite by its nature - the absolutely infinite, or substance, and the infinite in kind, or the attributes - and that which is infinite by the force of its cause - the affections of substance, or the modes. The second term of this distinction is then carried over as the first term of the next distinction: that which is infinite because it has no limits is distinguished from that which is infinite, because it is not determinable numerically, that which is unlimited or indefinite, even though included between a maximum and a minimum. For Macherey, these two distinctions also characterise that between reason (which is the adequate understanding of things) and the imagination (which involves the inadequate representation of things). Substance, or the absolutely infinite, can only be understood adequately and not imagined, and its affections, which are infinite by the force of their cause, can be understood adequately as infinite from the point of view of reason but only inadequately as unlimited or indefinite from the point of view of the imagination.

Macherey argues that "the traditional paradoxes of the infinite come from these distinctions not being respected". It is only necessary to re-establish these distinctions in order that "all the contradictions are not resolved, but effaced" (Macherey, 170). "For then", Spinoza argues,

they would have understood clearly what kind of Infinite cannot be divided into any parts, or cannot have any parts, and what kind of Infinite can, on the other hand, be divided into parts without contradiction. They would also have understood what kind of Infinite can be conceived to be greater than another Infinite, without any contradiction, and what kind cannot be so conceived (Let. XII). 
The presentation of these distinctions as contradictions depends only on the presentation of a problem whose terms have been inadequately distinguished and thus inadequately posed.

Rather than presenting the bad infinite and the actual infinite as contradictory, the latter being the result of the negation of the former, Macherey argues that the bad infinite, or what he refers to as the unlimited or indefinite, and the actual infinite, are one and the same thing as perceived respectively from the point of view of the imagination and from that of reason.

Macherey argues that the adequate understanding of a thing, which grasps the thing according to or after its cause, must be understood "affirmatively, in the sense of the absolute affirmation of the nature of the thing" (172). It is an understanding which is thus not determined negatively by the negation of the bad infinite, as it is according to the Hegelian dialectical logic, but positively from the point of view of the immanent cause of a thing, which is substance in the form of its attributes. The actual infinite "expresses directly the immanent, and non-transitive, relation which ties substance and its affections, and which is known only by the understanding" (172). The bad infinite or unlimited is the inadequate idea of the way in which the actual infinite is expressed in a finite mode. According to Macherey, the actual infinite, as apprehended adequately in the modes, "is not different to that infinity constitutive of substance, but is formally the same"(173). The actual infinite is therefore the immanent expression in the affections, or the finite modes, of infinite substance, which is their cause.

The Adequate and the Inadequate Idea of the Actual Infinite.

In the text of the example of Letter XII, the introductory sentence which Macherey translates similarly to Hegel as "All the inequalities of the space interposed between two circles [toutes les inégalités de l'espace interposé entre deux cercles]" (164), the reference to "the space interposed" between the two non-concentric circles actually "designates the set of distances" (164), that is, all of the different distances, which separate the two circumferences, including the two extreme distances, represented by the segments $\mathrm{AB}$ and $\mathrm{CD}$. The "inequalities of the space" should then be understood to refer to "the set of the differences between these unequal distances", or, what Macherey emphasises as "the variation" of these "differences", which is determined by the rotation of the segments from $A B$ towards $C D$, "in the sense of the hands of a watch" (165). This set, which is "the sum of the inequalities of distance included in this ... total space" (165), is a continuous and therefore infinite variation.

Rather than being limited, because included between a maximum (the circumference of circle A) and a minimum (that of circle B) and therefore being finite, as Hegel seems to indicate, the "inequalities of the space", or the differences between the unequal distances, and the "variation" of these 
differences, are only limited by the difference between the maximum distance, $\mathrm{AB}$, and the minimum distance, $\mathrm{CD}$.

It is this idea of the variation of differences which depends on the fact that the two circles are non-concentric, which is neglected by Hegel in his account of the geometrical example. For Macherey, the actual infinite is expressed in the example by the infinite variation of differences. The infinite variation can be understood as actually infinite from the point of view of reason, insofar as it has its cause in substance, and as indefinite or unlimited from the point of view of the imagination, insofar as it is represented as the sum of the multiplicity of its parts, since it is only from the point of view of the imagination that number can be applied to it.

According to Macherey, and contrary to Hegel, Spinoza's example does not function to represent the mediation between the different infinities that it represents, but rather it represents both infinities as existing simultaneously, their difference not being a matter of one's negation of the other, as embodied in an opposition or contradiction, but rather a matter of a difference in the degree of understanding, that is, an epistemological difference, which can vary from an inadequate idea of the way in which the actual infinite is expressed in the finite (as unlimited or indefinite), to the adequate understanding of the expression of the actual infinite in the finite, as the substantial cause of the finite, insofar as substance is the immanent cause of all finite things. The idea of infinite variation in the geometric example is therefore conceived by Macherey as inadequate when the infinite variation of differences is represented by the imagination as unlimited or indefinite, and as adequate when the infinite variation of differences is understood adequately as the actual infinite expressed in the finite, that is, infinite substance as expressed in its finite modes. Macherey can therefore conclude that "it seems ... the error of the imagination consists in taking as infinite, in the attempt to determine it numerically, a thing which is ... finite" (170).

The adequate expression of the actual infinite in the finite is, according to Macherey, the adequate knowledge of its immanent cause in substance. When the distinctions between the different infinities introduced by Spinoza in the discussion of "the problem of the Infinite" of Letter XII, are respected, Macherey argues that the contradictions involved in the relation of the finite to the infinite are not resolved, as Hegel considers them to have been by means of the dialectical logic of the negation of the negation, but, rather, they are effaced. The relation between the finite and the infinite is no longer one of contradiction. As Gueroult argues, "the solution to the problem of the infinite, due to [an adequate] idea of substance, puts an end to all antinomies" (209).

The adequate idea of the actual infinite, as the positive immanent expression of the absolutely infinite in the finite, is the adequate understanding of its implication in this relation. The inadequate idea of the actual infinite, as 
unlimited or indefinite, is not in a relation of negation to this positive expression of the infinite, but is rather only an imaginary representation of the actual infinite, and, as such, it betrays only a partial degree of understanding.

\section{The Letter on the Infinite}

Having utilised the geometrical example of Spinoza's Letter XII to distinguish the respective interpretations of Spinoza by Hegel and Macherey, Deleuze's reading of Spinoza will be distinguished from that of Hegel and Macherey insofar as it too offers an alternative reading of what Deleuze describes as the "very bizarre, curious" geometrical example. By implicating Leibniz's understanding of the early form of the infinitesimal calculus in his reading of the geometrical example, Deleuze argues that it is able to be characterised as an example of what had already been established of the infinitesimal calculus. He thereby traces an alternative lineage between Leibniz and Spinoza to that determined by Hegel in the development of the dialectical logic. It is by means of Deleuze's reading of Letter XII that the investigation into the logic that is mobilised in his reading of Spinoza in Expressionism in Philosophy, as an alternative to the dialectical logic, is developed in The Logic of Expression: Quality, Quantity andIintensity in Spinoza, Hegel and Deleuze, ${ }^{12}$ and it is Gueroult's reading of Letter XII which provides the point of departure for this investigation. However, it is the explication of this point of departure that remains the focus of the present paper.

In "Spinoza's Letter on the Infinite", Gueroult suggests that the meaning of the geometrical example is "generally wrongly understood", and that it has been "vitiated in its principle through errors in translation" (206). Macherey continues this argument in Hegel ou Spinoza when he writes that "the example as reproduced by Hegel is not the same as that given in the text of Spinoza". $\mathrm{He}$ argues that the same example is exploited by each commentator "in markedly different ways" (168). Macherey and Gueroult come to very similar conclusions in relation to their respective interpretations of Letter XII and of Hegel's misreading of it. However, Macherey criticises Gueroult's fourfold distinction of the definitions of infinity in Letter XII: "In his commentary on the letter on infinity, Gueroult enumerates four successive cases which, in relation to the text of Spinoza, seems excessive" (169). Gueroult breaks the distinctions down as follows:

1. The thing infinite by its essence or by virtue of its definition.

2. The thing without limits, not by virtue of its essence, but by virtue of its cause.

3. The thing infinite insofar as without limits.

4. The thing infinite insofar as its parts, although included within a maximum and minimum known to us, cannot be expressed by any number. (183) 
Macherey considers the third definition to be superfluous since when understood from the point of view of reason, it is the same as the second definition, and when the second definition is represented inadequately from the point of view of the imagination, the result is the third definition. The significance of this move is that the fourth definition, which is illustrated by the geometrical example, then becomes the example of the implied difference in the second definition, between an imaginary and a rational understanding of the infinite. For Gueroult, however, the significance of the third definition rests, on the one hand, on its distinction from the fourth definition and, on the other hand, on its importance as to his criticism of the Hegelian interpretation of Letter XII. Presenting his case, Gueroult argues that the geometrical example, which illustrates the fourth definition, "is advanced in order to refute the false interpretation" that "every infinite is such that its magnitude is so excessive ... that we cannot perceive its limits, or that its variations are not contained between any boundaries" (202). This explanation merely repeats the third definition. To claim that the infinite must everywhere be said to be inferred from the multitude of its parts is to explain the infinite by the finite, which can only then "be avoided by the negation of the Infinite on behalf of the finite" (192). Gueroult is critical of Hegel's reading of Letter XII insofar as it relies on the third definition in order to secure the mediation of the dialectical relation between the finite and the infinite. Macherey's recognition of this is the reason that he considers the third definition superfluous to an adequate understanding of Spinoza's infinite. Gueroult argues that, contrary to Hegel's interpretation, the third and fourth definitions present two different definitions of the infinite which do not implicate each other in a dialectical relation of negation. What Hegel presents as a dialectical relation of opposition between a mathematical and a philosophic infinite, Gueroult presents as a difference between a mathematical infinite, that is, an arithmetic or algebraic infinite, as defined by the third definition, ${ }^{13}$ and a geometric infinite, as illustrated by the fourth definition. ${ }^{14}$ Hegel's reliance on the third definition renders the geometric infinite, as characterised by Spinoza in the geometrical example, superfluous to his interpretation of Letter XII. Hegel implicates the mathematical infinite solely as an algebraic infinite. Spinoza's depreciation of arithmetic, which, along with number, is reduced to a finite being of the imagination,,$^{15}$ is set against what Gueroult describes as Spinoza's "correlative exaltation of geometrical thought" (206). What Hegel recognises as Spinoza's rejection of the mathematical infinite is rather a rejection of the algebraic infinite in the name of a geometric infinite. Spinoza's geometric infinite is therefore different to Hegel's mathematical algebraic infinite. Gueroult suggests that "this ... establishes a vigorous contrast between the mathematical philosophy of Spinoza and those of the philosophers of his time" (201), a contrast which could well be extended to include the interpretations of both Macherey and 
Hegel. Spinoza here sets geometry against algebra, a properly geometric infinite against an algebraic infinite. Gueroult suggests that this "opens an abyss between arithmetic and geometry" (201), which he then maps onto the epistemological difference as the difference between an adequate understanding of the geometric infinite as actually infinite from the point of view of reason, and an inadequate representation of this geometric infinite as an algebraic infinite from the point of view of the imagination.

The Geometric Infinite as "an Infinite Sum of Differentials".

Deleuze's interpretation of Letter XII in Expressionism in Philosophy takes up this distinction between algebra and geometry in order to redeploy the definitions of the infinite from the point of view of the geometrical example. Deleuze elicits the support of Leibniz to argue that Spinoza's exposition of the geometric infinite in Letter XII goes "further on this point than many mathematicians" (EPS 203). According to Deleuze, the properly geometric infinite characterised in the fourth definition, as distinct from the algebraic infinite of the third definition, defines "a strictly extensive infinity", which is a properly "modal and quantitative" infinite (EPS 202). A thorough analysis of Deleuze's interpretation of Letter XII is required to illustrate how he comes to this conclusion.

Deleuze distinguishes his interpretation of Letter XII from that of Hegel by arguing that "two concentric circles of which one is interior to the other lack the greatest and smallest distance of one circle to the other" ${ }^{16}$ In order to explicate what happens across this maximum and minimum orthogonal distance, Deleuze offers a translation of the sentence with which Spinoza introduces the example. Spinoza writes: "omnes inæqualitates spatii duobus circulis $\mathrm{AB}, \& \mathrm{CD}$, interpositi". ${ }^{17}$ Deleuze translates this as "the sum of the inequalities of distance included between two circles, $\mathrm{AB}$ and CD". ${ }^{18}$ Deleuze considers this example to define "a very particular geometric infinite" ${ }^{19}$ Hegel considers only the algebraic infinity of unequal distances, that is, if all the lines or segments that go from one circle to the other, the orthogonal distances, are placed end to end, the sum would be infinite. This sum

would be equally infinite if the two circles were concentric and if all the segments ... were equal, it is [therefore] clear that [for Hegel] the infinite sum of the unequal segments ... is not related to their inequality and is not delimited by the maximum and the minimum of their variations"(Gueroult, 207).

Deleuze, on the contrary, considers it to be a question, not of the sum of the segments, or orthogonal distances, but rather of the sum of their inequalities, that is, the sum of the differences between the orthogonal distances.

Macherey also distinguishes his reading of Letter XII from that of Hegel in this way, however, he does not conceive of the infinite variation of differences, understood adequately from the point of view of reason, to be a geometric infinite. Macherey reserves the use of the term "sum" for references associated 
with the inadequate representation of this infinite from the point of view of the imagination. An infinite sum which is only inadequately supposed to be infinite, or unlimited, by the multitude of its parts, is an algebraic infinite. Deleuze considers the adequate understanding of the infinite from the point of view of reason to be an infinite sum which is distinctly geometrical. He argues that the geometric infinite "is not strictly speaking 'unlimited': for it relates to $\ldots$ the ... maximum $(\mathrm{AB})$ and $\ldots$ minimum $(\mathrm{CD})$ distance between two nonconcentric circles, and these distances attach to a perfectly limited and determinate space" (EPS 202). When the orthogonal distances vary continuously from $\mathrm{AB}$ to $\mathrm{CD}$, the sum of the successive differences is not simply a matter of "an infinite sum of finite quantities", as Hegel contends, nor is it "the infinite variation of differences" as Macherey contends, but, Gueroult argues, and Deleuze backs up this argument, it should rather be understood as "an infinite sum of differentials" (207).

The concept of the differential was introduced by developments in the infinitesimal calculus during the later part of the seventeenth century. Deleuze positions Spinoza's geometric infinite within the early stages of this development, which Carl Boyer, in The History of the Calculus and its Conceptual Development, describes as being "bound up with concepts of geometry $\ldots$ and with explanations of $\ldots$ the infinitely small". ${ }^{20}$ Boyer describes the infinitesimal calculus as dealing with "the infinite sequences ... obtained by continuing ... to diminish ad infinitum the intervals between the values of the independent variable. ... By means of [these] successive subdivisions ... the smallest possible intervals or differentials" are obtained (ibid., p. 12). The differential can therefore be understood to be the infinitesimal difference between consecutive values of a continuously diminishing quantity. Boyer refers to this early form of the infinitesimal calculus as the infinitesimal calculus from "the differential point of view" (12).

Gueroult provides a succinct definition of the differential in relation to the geometrical example of Letter XII when he suggests that the determinate distance ' $A B$ minus $C D$ ' "includes an actual infinity of infinitely small distances, and consequently is indivisible into discontinuous parts" (Gueroult 209). Each of these infinitely small distances corresponds to a differential. Boyer argues that, "the derivative would in this case be defined as the quotient of two such differentials, and the integral would then be the sum of a number (perhaps finite, perhaps infinite) of such differentials" (Boyer, 12). The geometric infinite of Spinoza's Letter XII, as "an infinite sum of differentials", can therefore be understood to be an example of an integral from the differential point of view.

Gueroult identifies the integral in the geometrical example as a definite integral when he argues that "the distance $\mathrm{CD}$ is a minimal quantity obtained by continuously diminishing the infinitely small parts of $\mathrm{AB}$, the sum of these 
diminishments being a definite integral, that is, a finite quantity ... resulting from an infinite summation of differentials" (209). According to Gueroult, the infinite sum of the differentials is a definite integral, and a definite integral is a finite quantity. In this way, the geometrical example illustrates the implication and envelopment of the infinite in the finite.

What Deleuze then does with the concept of the infinitesimal calculus from the differential point of view distinguishes his interpretation of Letter XII from that of Gueroult. Whereas Gueroult only suggests the implication of the infinitesimal calculus in his interpretation of Spinoza's Letter XII, Deleuze explores this implication in his interpretation of Spinoza's work as a whole. It is here that we begin to approach not only the originality of Deleuze's interpretation of Spinoza, but also one of the points where Deleuze departs from Spinozism by tracing an alternative lineage in the history of philosophy in relation to Leibniz.

\section{The Differential Point of View}

The infinitesimal calculus consists of two branches which are inverse operations: differential calculus, which is concerned with calculating derivatives, or differential relations; and integral calculus, which is concerned with integration, or the calculation of the infinite sum of the differentials. The derivative, from the differential point of view of the infinitesimal calculus, is the quotient of two differentials, that is, a differential relation, of the type $d y / d x$. The differential, $d y$, is an infinitely small quantity, or what Deleuze describes as "a vanishing quantity" ${ }^{\prime 2}$; a quantity smaller than any given or givable quantity. Therefore, as a vanishing quantity, $d y$, in relation to $y$, is, strictly speaking, equal to zero; as is $d x$, in relation to $x$. Given that $y$ is a quantity of the abscissa, and that $x$ is a quantity of the ordinate, $d y=0$ in relation to the abscissa, and $d x=0$ in relation to the ordinate. The differential relation can therefore be written as $d y / d x=0 / 0$. However, despite the fact that $d y / d x=0 / 0$, and that $0 / 0=0$, the relation between these two differentials, $d y / d x$, does not equal zero, $d y / d x \uparrow 0$. This is because the differentials do actually exist as vanishing quantities, that is, despite the fact that, strictly speaking, they equal zero, they are still not yet, or not quite equal to, zero. "What subsists when $d y$ and $d x$ cancel out under the form of vanishing quantities is the relation $d y / d x$ itself" (17.2.81). It is here that Deleuze considers seventeenth century logic to have made "a fundamental leap", by determining "a logic of relations" (10.3.81). He argues that "under this form of infinitesimal calculus is discovered a domain where the relations no longer depend on their terms". According to Deleuze, "the terms between which the relation establishes itself are neither determined, nor determinable. Only the relation between its terms is determined" (10.3.81). This is the logic of relations that Deleuze locates in the infinitesimal calculus of the seventeenth century, which he then mobilises in his reading of Spinoza's Letter XII, and in his 
reading of Spinoza's work as a whole, particularly in relation to the physics of bodies in the second part of the Ethics (P13).

The differential relation, which Deleuze characterises as a "pure relation", because it is independent of its terms, and which subsists insofar as $d y / d x \uparrow 0$, has a perfectly expressible finite quantity designated by a third term, $z$, such that $d y / d x$ equals $z$. Deleuze argues that "when you have a [differential] relation derived from a circle, this relation doesn't involve the circle at all but refers [rather] to what is called a tangent" (17.2.81). A tangent is a line that touches a circle or curve at one point. The gradient of a tangent indicates the rate of change of the curve at that point, that is, the rate at which the curve changes on the $y$-axis relative to the $x$-axis. The differential relation therefore serves in the determination of this third term, $z$, the value of which is the gradient of the tangent to the circle or curve. In the case of the geometrical example, it is the tangents to the circumferences of the two circles at $\mathrm{A}$ and $\mathrm{C}$ which define the maximum and minimum limits of the orthogonal distances $\mathrm{AB}$ and $\mathrm{CD}$.

When referring to the geometrical study of curves in his early mathematical manuscripts, Leibniz writes that "the differential calculus could be employed with diagrams in an even more wonderfully simple manner than it was with numbers". ${ }^{22}$ Leibniz presents one such diagram in a paper entitled "Justification of the Infinitesimal Calculus by That of Ordinary Algebra", ${ }^{23}$ when he offers an example of what had already been established of the infinitesimal calculus in relation to particular problems before the greater generality of its methods were developed. An outline of the example that Leibniz gives is as follows:

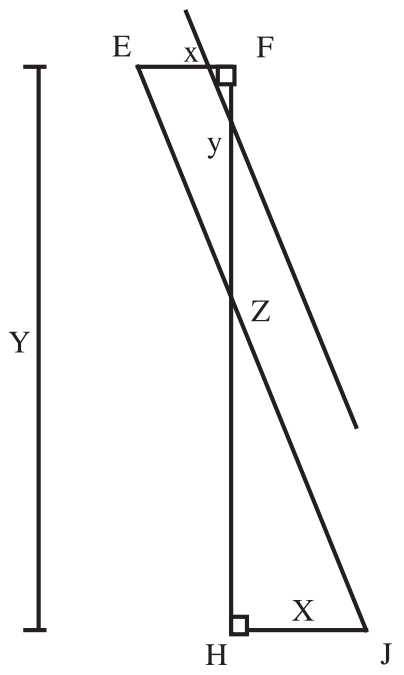

Figure 2 
Since the two right triangles, ZFE and ZHJ, that meet at their apex, point Z, are similar, it follows that the ratio $y / x$ is equal to $(Y-y) / X$. As the straight line EJ approaches point $F$, maintaining the same angle at the variable point $Z$, the lengths of the straight lines FZ and FE, or $y$ and $x$, steadily diminish, yet the ratio of $y$ to $x$ remains constant. When the straight line EJ passes through $\mathrm{F}$, the points $\mathrm{E}$ and $\mathrm{Z}$ coincide with $\mathrm{F}$, and the straight lines, $y$ and $x$, vanish. Yet $y$ and $x$ will not be absolutely nothing since they preserve the ratio of $\mathrm{ZH}$ to $\mathrm{HJ}$, represented by the proportion $(Y-y) / X$, which in this case reduces to $Y / X$, and obviously does not equal zero. The relation $y / x$ continues to exist even though the terms have vanished, since the relation is determinable as equal to $Y / X$. In this algebraic calculus, the vanished lines $x$ and $y$ are not taken for zeros since they still have an algebraic relation to each other. "And so", Leibniz argues, "they are treated as infinitesimals, exactly as one of the elements which ... differential calculus recognises in the ordinates of curves for momentary increments and decrements"(545). That is, the vanished lines $x$ and $y$ are determinable in relation to each other only insofar as they can be replaced by the infinitesimals $d y$ and $d x$, by making the supposition that the ratio $y / x$ is equal to the ratio of the infinitesimals, $d y / d x$. In the first published account of the calculus, Leibniz defines the ratio of infinitesimals as the quotient of firstorder differentials, or the differential relation. He says that "the differential $d x$ of the abscissa $x$ is an arbitrary quantity, and that the differential $d y$ of the ordinate $y$ is defined as the quantity which is to $d x$ as the ratio of the ordinate to the subtangent" (Boyer, 210). Leibniz considers differentials to be the fundamental concepts of the infinitesimal calculus, the differential relation being defined in terms of these differentials.

Deleuze considers Spinoza's Letter XII to provide an example of what had already been established of the infinitesimal calculus in relation to a particular problem at the level of geometry; the geometric infinite. Spinoza's example is schematic in just the right proportion to be representable using this Leibnizian formulation of the early form of the infinitesimal calculus. The example of two non-concentric circles (Figure 1) can be transposed onto Leibniz's example (Figure 2) by letting $\mathrm{Z}$ be the point on the maximum orthogonal distance $\mathrm{AB}$ (Figure 3), such that $\mathrm{AZ}$ equals $\mathrm{AB}$ minus $\mathrm{CD}$. The points $\mathrm{A}$ and $\mathrm{Z}$ represent the points of tangency of the minimum and maximum orthogonal distances respectively on $A B$. Since the values of the differences of distance of Spinoza's example vary between A and Z, then, according to Leibniz's justification of the infinitesimal calculus by algebra, each of these differences of distance can be represented by an algebraic ratio of infinitesimals, that is, by a differential relation, $d y / d x$, the infinite sum of these differential relations being the infinite sum that Spinoza refers to in Letter XII. The geometric infinite, as "an infinite sum of differentials", can therefore be understood to be an example of an integral from the differential point of view of the 


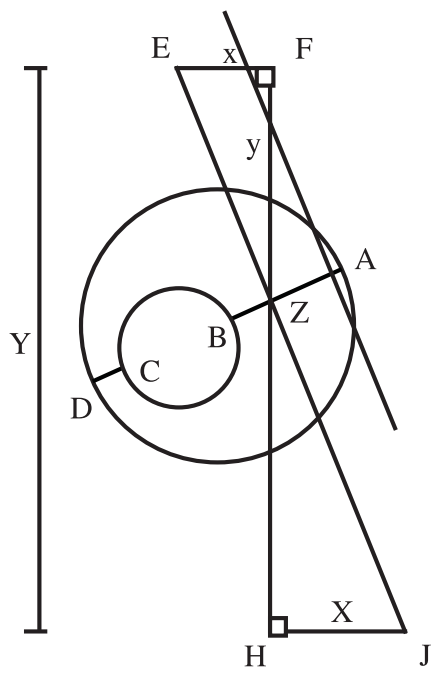

Figure 3

infinitesimal calculus. Insofar as this infinite sum is understood to function as such an integral, it provides an example of what Deleuze describes as the "integral calculus performed unknowingly" $(1994,174)$.

The differential point of view of the infinitesimal calculus approaches integration as a process of summation by considering the problem of finding the area under the graph of a function. This problem is dealt with by dividing up the area under the curve into a large number of rectangles. The area under the curve is the sum of the infinitely small and infinitely numerous rectangles. The difference between this sum and the actual area is considered small enough to be neglected. The integral is therefore the finite magnitude of the area.

\section{A New Theory of Relations}

Leibniz recognised integration to be a process not only of summation, but also of the inverse transformation of differentiation, so the integral is not only the sum of differentials, but also the inverse of the differential relation. In the early nineteenth century, the process of integration as a summation was overlooked by most mathematicians in favour of determining integration, instead, as the inverse transformation of differentiation. Hegel continued this tendency by defining the integral solely as the inverse of the differential relation. According to Hegel, "the integral calculus has been simplified and more correctly determined merely by the fact that it is no longer taken to be a 
method of summation in which it appeared essentially connected with the form of a series" (SL 294). The main reason for this was that by extending sums to an infinite number of terms, problems began to emerge if the series did not converge. The value or sum of an infinite series is only determinable if the series converges. Divergent series have no sum. It was considered that reckoning with divergent series would therefore lead to false results. However, the problem of integration as a process of summation from the differential point of view of the infinitesimal calculus did continue to be explored. It was Augustin Cauchy (1789-1857) who first introduced specific tests for the convergence of series, so that divergent series could henceforth be excluded from being used to try to solve problems of integration, because of their propensity to lead to false results. ${ }^{24}$ This method was later reformulated by Cauchy as the determination of the definite integral, which is distinguished from the indefinite integral only insofar as the definite integral is used to determine the limit of the sum of the area under the curve between two points on the $\underline{x}$-axis, whereas the indefinite integral, as the primitive function whose derivative is the given function, determines the whole curve. Both integrals, definite and indefinite, are determinable by the inverse transformation of the differential relation; the definite integral being determined within a limited interval. Whereas Gueroult equates the infinite sum of Spinoza's example with this definite integral, Deleuze rather appeals to the "barbaric or pre-scientific interpretations of the differential calculus", ${ }^{25}$ as presented by the differential point of view of the infinitesimal calculus, according to which integration is a method of summation in the form of a series.

The object of the process of integration in general is to determine from the coefficients of the given function of the differential relation the original function from which they were derived. Put simply, given a relation between two differentials, $d y / d x$, the problem of integration is how to find a relation between the quantities themselves, $y$ and $x$. This problem corresponds to the geometrical method of finding the function of a curve characterised by a given property of its tangent. The differential relation is thought of as another function which describes, at each point on an original function, the gradient of the line tangent to the curve at that point. The value of this "gradient" indicates a specific quality of the original function; its rate of change at that point. The differential relation therefore indicates the specific qualitative nature of the original function at the different points of the curve.

The inverse process of this method is differentiation, which, in geometrical terms, determines the differential relation as the function of the line tangent to a given curve. Put simply, to determine the tangent of a curve at a specified point, a second point that satisfies the function of the curve is selected, and the gradient of the line that runs through both of these points is calculated. As the second point approaches the point of tangency, the gradient of the line between 
the two points approaches the gradient of the tangent. The gradient of the tangent is, therefore, the limit of the gradient of the line between the two points.

It was Newton who first came up with this concept of a limit. He conceptualized the tangent geometrically, as the limit of a sequence of lines between two points on a curve, which he called a secant. As the distance between the points approached zero, the secants became progressively smaller, however they always retained "a real length". The secant therefore approached the tangent without reaching it. When this distance "got arbitrarily small (but remained a real number)", ${ }^{26}$ it was considered insignificant for practical purpose, and was ignored. What is different in Leibniz's method is that he "hypothesized infinitely small numbers - infinitesimals - to designate the size of infinitely small intervals" (ibid). For Newton, on the contrary, these intervals remained only small, and therefore real. When performing calculations, however, both approaches yielded the same results. But they differed ontologically, because Leibniz had hypothesized a new kind of number, a number Newton did not need since "his secants always had a real length, while Leibniz's had an infinitesimal length" (ibid).

For the next two hundred years, various attempts were made to find a rigorous arithmetic foundation for the calculus; one that did not rely on either the mathematical intuition of geometry, with its tangents and secants, which was perceived as imprecise, because its conception of limits was not properly understood, or on the vagaries of the infinitesimal, which made many mathematicians wary, so much so that they refused the hypothesis outright, despite the fact that Leibniz "could do calculus using arithmetic without geometry - by using infinitesimal numbers"(ibid). It was not until the late nineteenth century, that an adequate solution to this problem of rigor was posed. It was Karl Weierstrass (1815-1897) who "developed a pure nongeometric arithmetization for Newtonian calculus" (ibid 230), which provided the rigor that had been lacking. "Weierstrass's theory was an updated version of Cauchy's earlier account" (ibid 309), which had also had problems conceptualising limits. Cauchy actually begs the question of the concept of limit in his proof. ${ }^{27}$ In order to overcome this problem of conceptualising limits, Weierstrass "sought to eliminate all geometry from the study of ... derivatives and integrals in calculus". ${ }^{28}$ In order to characterize calculus purely in terms of arithmetic, it was necessary for the idea of a function, as a curve in the Cartesian plane defined in terms of the motion of a point, to be completely replaced with the idea of a function that is, rather, a set of ordered pairs of real numbers. The geometric idea of 'approaching a limit' had to be replaced by an arithmetized concept of limit that relied on static logical constraints on numbers alone. This approach is commonly referred to as the epsilon-delta method. Deleuze argues that "it is Weierstrass who bypasses all the 
interpretations of the differential calculus from Leibniz to Lagrange, by saying that it has nothing to do with a process. ... Weierstrass gives an interpretation of the differential and infinitesimal calculus which he himself calls static, where there is no longer fluctuation towards a limit, nor any idea of threshold" (22.2.72). The calculus was thereby reformulated without either geometric secants and tangents or infinitesimals; only the real numbers were used.

Because there is no reference to infinitesimals in this Weierstrassian definition of the calculus, the designation 'the infinitesimal calculus' was considered to be "inapproporiate". ${ }^{29}$ Weierstrass's work not only effectively removed any remnants of geometry from what was now referred to as the differential calculus, but it eliminated the use of Leibnizian-inspired infinitesimal arithmetic in doing the calculus for over half a century. It was not until the late 1960's, with the development of the controversial axioms of nonstandard analysis by Abraham Robinson (1918-1974), that the infinitesimal calculus was given a rigorous foundation, and a formal theory of the infinitesimal calculus was constructed, thus allowing Leibniz' ideas to be "fully vindicated", ${ }^{30}$ as Newton's had been thanks to Weierstrass. It is specifically in relation to these developments that Deleuze's appeal to the "barbaric or pre-scientific interpretations of the differential calculus", should be understood.

Although it is only later in the development of the infinitesimal calculus that the tangent comes to be considered as a limit, and that the differential relation is used to calculate 'limits,' Deleuze contends that the maximum and minimum illustrated in Spinoza's geometrical example are suggestive of such limits. He introduces the concepts of the differential relation and limits into his interpretation not only of Letter XII, but also into his interpretation of the physics of bodies presented in the second part of the Ethics. So, according to Deleuze, the value of $z$, which was determined by Leibniz in relation to the differential relation $(d y / d x)$ as the gradient of the tangent, functions as a limit. When the relation establishes itself between infinitely small terms, it does not cancel itself out with its terms, but rather tends towards a limit, $z$. Since the differential relation approaches closer to its limit as the differentials decrease in size, or approach zero, the limit of the relation is represented by the relation between the infinitely small. Of course, despite the geometrical nature of the idea of a variable and a limit, the former 'decrease in size' or 'approach zero', and the differential relation 'approaches' or 'tends towards' the latter, they are not essentially dynamic, but involve purely static considerations, that is, they are rather "to be taken automatically as a kind of shorthand for the corresponding developments of the epsilon-delta approach" (ibid 277). It is in this sense that the differential relation between the infinitely small refers to something finite. Or, as Deleuze suggests, it is in the finite itself that there is the "mutual immanence" (17.2.81) of the relation and the infinitely small. 
Given that the method of integration provides a way of working back from the differential relation, the problem of integration is, therefore, how to reverse this process of differentiation. This can be solved by determining the inverse of the given differential relation according to the inverse transformation of differentiation, as Hegel had favoured. Or, a solution can be determined from the differential point of view of the infinitesimal calculus by considering integration as a process of summation in the form of a series, according to which, given the specific qualitative nature of a tangent at a point, the problem becomes that of finding, not just one other point determinative of the differential relation, but a sequence of points, all of which together satisfy, or generate, a curve and therefore a function in the neighbourhood of the given point of tangency, which therefore functions as the limit of the function.

Deleuze considers this to be the base of the infinitesimal calculus as understood or interpreted in the seventeenth century. The formula for the problem of the infinite that Deleuze extracts from the geometrical example of Letter XII, by means of this seventeenth-century understanding of the infinitesimal calculus, is that "something finite consists of an infinity under a certain relation". Deleuze considers this formula to mark "an equilibrium point, for seventeenth-century thought, between the infinite and the finite, by means of a new theory of relations" (17.2.81).

By implicating Leibniz's understanding of the early form of the infinitesimal calculus in his interpretation of the geometrical example of Spinoza's Letter XII, Deleuze not only demonstrates how Spinoza eludes the grasp of the dialectical progression of the history of philosophy, but also nominates Leibniz as one of the figures with whom he engages in his project of renewing the history of philosophy by constructing an alternative lineage in the history of philosophy. Deleuze reads Spinoza's geometrical example as providing an example of what had already been established by the infinitesimal calculus in response to the problem of the infinite. He considers seventeenth century thought, and this includes Spinoza, to have developed a new theory of relations by means of the infinitesimal calculus, one which is determined according to the differential point of view of the infinitesimal calculus. The infinitesimal calculus puts into play a certain type of relation for Deleuze - the differential relation - which is characteristic of the compositional relations between individuals. It is by exploiting the implications of the differential point of view of the infinitesimal calculus in his interpretation of the physics of bodies in the second part of the Ethics, that Deleuze determines the logic that is mobilised in his reading of the system of the Ethics as a whole. This strategy of reading the Ethics marks not only the originality of Deleuze's interpretation of Spinoza, but also one of the points where Deleuze can be considered to depart from the Cartesian and Hegelian Spinoza familiar to scholars working in the field of Spinoza studies by tracing 
an alternative lineage in the history of philosophy between Spinoza's ontology and the mathematics of Leibniz.

\section{University of Queensland}

\section{References}

1. P. Macherey, Hegel ou Spinoza, Paris: Presses Universitaires de France, 1979, translations from this text are mine; from now on referred to as Macherey in the text.

2. Gilles Deleuze, Expressionism in Philosophy, Spinoza, trans. M. Joughin, New York: Zone Books 1992, from now on referred to as EPS in the text.

3. Georg Wilhelm Friedrich Hegel, Science of Logic, trans. A.V. Miller, London: George Allen \& Unwin 1969, p. 251.

4. B. Spinoza, "Letter XII", in: The Collected Works of Spinoza Volume I, ed. and trans. E. Curley, New Jersey: Princeton University Press 1985, p. 201-205, from now on referred to as Let. XII.

5. G.W.F. Hegel, Lectures on the History of Philosophy, Volume 3, London: Routledge 1955, p. 262, from now on referred to as LHP.

6. Hegel, Science of Logic, p. 250.

7. Spinoza, Ethics, in: op.cit., p. 28.

8. Hegel, Science of Logic, p. 250.

9. M. Gueroult, "Spinoza's Letter on the Infinite", in: Spinoza: A Collection of Critical Essays, Indiana: University of Notre Dame Press 1973, p. 190.

10. Gueroult, op. cit., p. 207.

11. Gilles Deleuze, Seminars: 20 January 1981, trans. S. Duffy; URL http://www.webdeleuze.com.

12. Simon Duffy, The Logic of Expression: Quality, Quantity and Intensity in Spinoza, Hegel and Deleuze, Aldershot: Ashgate 2006.

13. Dedron and Itard offer the following definition of the mathematical infinite: "the idea of an infinite number is merely an abstract idea which expresses an imagined limit which no finite number can ever reach". Whether the infinite approaches the infinitely small, insofar as formed, for example, by the series of fractions " $1 / 2,1 / 4,1 / 8,1 / 16, \ldots$, each term being half the previous one", whose sum, if continued to infinity would be equal to 1 , the number 1 being "the limit of the sum of the series"; or the infinitely large, insofar as formed, for example, by "the series $2,4,6,8,16 \ldots$, or any other increasing series $\ldots$ the more terms of the series ... the greater is their sum, and this sum can exceed any number however large" (P. Dedron, and J. Itard, Mathematics and Mathematicians. Vol. 2, trans. J.V. Field, London: Transworld 1974, p. 297).

14. Pierre Dedron and Jean Itard put forward the argument that geometry is able to "attach clear, simple and precise meanings to expressions which make use of the term or the idea of infinity"(op. cit., 298).

15. Cf. "Measure, Time, and Number are nothing but Modes [...] of imagining"(Let. XII).

16. Deleuze, Seminars, 20.01.81.

17. B. Spinoza, Spinoza Opera. ed. Carl Gebhardt, Heidelberg: Carl Winters Univeritätsbuchhandlung 1925 , p. 59.

18. This is my translation from the French: "la somme des inégalités de distances comprises entre deux cercles", (Deleuze, Spinoza et le problème de l'Expression, Paris: Editions de Minuit 1986, p. 184), which, I contend, is mistranslated by Martin Joughin as "the sum of the unequal distances between two ... circles" (EPS 202).

19. Deleuze, Seminars, 20.01.81

20. C. Boyer, The History of the Calculus and its Conceptual Development, New York: Dover Publications 1959, p. 11.

21. Deleuze, Seminars, 17.2.81, in the following referred to by the date in the text. 
22. G.W. Leibniz, The Early Mathematical Manuscripts, trans. M.J. Childs, Open Court 1920, p 53.

23. G.W. Leibniz, "Letter to Varignon, with a Note on the 'Justification of the Infinitesimal Calculus by that of Ordinary Algebra"', Philosophical Papers and Letters, Dordrecht: D. Reidel 1969, p. 545; see also G. Deleuze, The Fold: Leibniz and the Baroque trans. T. Conley, Minneapolis: University of Minnesota Press 1993, p. 18.

24. See: Boyer, The History of the Calculus ..., p. 287.

25. G. Deleuze, Difference and Repetition, trans. P. Patton, New York: Columbia University Press 1994, p. 171.

26. G. Lakoff, and R. Nafiez, Where Mathematics Comes From: How the Embodied Mind Brings Mathematics into Being, New York: Basic Books 2000, p. 224.

27. See: Boyer, The History of the Calculus ..., p. 281.

28. G. Lakoff, and R. Nafiez, op. cit., p. 309

29. See: Boyer, The History of the Calculus ..., p. 287.

30. A. Robinson, Non-Standard Analysis, New Jersey: Princeton University Press 1996, p. 2. 